\title{
Contribution à l'Etude de Bathynella d'Europe: Bathynella natans Vejdovsky, un dilemme à résoudre
}

\author{
Par Eugène Serban ${ }^{1}$ )
}

Avec planches 29 (1) - 35 (7)

\begin{abstract}
«Je ne puis m'empêcher d'«aller voir» chaque fois que cela m'est possible. Et quand j'ai vu, je revois, puis je regarde encore, car une douloureuse expérience m'a appris combien il est facile de se tromper».

E. G. Racovitza
\end{abstract}

Découverte, étudiée et décrite à Prague par Vejdovsky (1882), située parmi les Syncarides par Calman (1899) et retrouvée après trente ans à Bâle par Chappuis (1914), la Bathynella a fait époque.

A l'espèce type B.natans Vejd., Delachaux (1919) ajoute une seconde, B. chappuisi, capture d'une troisième station, la Grotte de Ver (Suisse).

Chaque redécouverte de ce «fossile vivant» donne naissance à une nouvelle note, les deux espèces étant citées à tour de rôle par différents auteurs. L'aire géographique étendue, la fréquence de sa rencontre, anéantissent complètement l'idée qu'on s'était faite de sa rareté.

Probablement par une coïncidence, l'année 1954, qui donne jour à plusieurs travaux sur ce sujet, représente un moment décisif pour la systématique de Bathynella.

Karaman (1954) dans son «Über die Bathynelliden Jugoslawiens» reprend les recherches commencées en 1934, en aboutissant à des conclusions qui, à vrai dire, ne confirment guère l'idée de l'existence des deux espèces. ${ }^{2}$ )

1) Institutul de Speologie, Str. Dr. Capşa 8, Bucureşti, Romania.

$\left.{ }^{2}\right)$,Es zeigte sich nun, wie auch damals, daß tatsächlich unsere Tiere teils zu natans und teils zu chappuisi zuzurechnen sind, daß aber diese zwei Arten nicht weiter als selbständige Arten zu führen wären. Beiden zahlreichen in letzter Zeit aufgestellten Arten, Unterarten und Formen kommen die Charaktere beider Arten so vermengt vor, daß sie für systematische Fragen unbrauchbar wurden" (Karaman, 1954, p. 69/1). 
Bien que Delachaux ait pu établir dix caractéristiques morphologiques séparant B.chappuisi de B.natans, la découverte d'individus à traits intermédiaires, soulève la question de la valeur des deux espèces.

Delamare Deboutteville et Chappuis (1954), ne possédant pas toutes les données pouvant confirmer l'existence des deux espèces, laissent cette question en suspens: «... il existe en Europe»-disent-ils «deux groupes de Bathynella, d'une part le complexe natanschappuisi, d'autre part l'espèce vandeli. Il n'est pas possible de pousser plus loin le travail pour le moment.» ${ }^{3}$ ) Il est connu que les auteurs attribuent les captures de France et d'Espagne à l'une des deux espèces, leur conférant le qualificatif de forme, parce que: «... il aurait été imprudent de donner aux formes décrites le statut d'espèces nouvelles. Il apparaît en effet que nos connaissances sont encore beaucoup trop imparfaites pour qu'il soit possible de les classer avec certitude, ce qui est le but de toute systématique». ${ }^{3}$ )

La même année Jakobi arrive à une surprenante conclusion en ce qui concerne les deux espèces. Les recherches portant sur un nombre considérable d'exemplaires appartenant à plusieurs populations d'Allemagne, ont eu comme principal résultat l'opinion que B.chappuisi Delachaux est synonyme de B.natans Vejdovsky. Dans le mème ouvrage, en se basant sur la structure de la partie masticatrice de la mandibule, Jakobi montre qu'en Allemagne B.natans est représentée par quatre races, assez nettement isolées du point du vue territorial. Ainsi, les incertitudes des différents auteurs sur l'existence de deux espèces sont résolues à la mème époque par Jakobi, qui de cette façon introduit aussi une vision moderne de la systématique du groupe. En se rapportant au matériel de Vejdovsky, dont il ne pouvait faire abstraction, le spécialiste allemand précise: »Die Mandibelform, die Vejdorsky 1882 von Prager zeichnet, kann nicht für einen Vergleich mit den oben angeführten Formen verwendet werden. Jedoch kann man sagen, daß die Bezahnung mit keiner der bisher bekannten Bezahnungsweisen übereinstimmt (B.natans natans). $\left.\otimes^{4}\right)$

C'est toujours à partir de 1954 qu'en Tchécoslovaquie paraissent plusieurs notes contenant des informations sur les populations de la «terra typica».

3) Delamare Deboutteville et Chappuis, 1954, p. 54.

4) Jakobi, 1954, p. 53. 
Sterba (1954), le premier, annonce la présence de B.chappuisi dans les puits de Brünn, puis une année plus tard dans la grotte Blaska (à l'Est des Monts Tatra). En tenant compte des résultats de Jakobi (1954), dans sa note "Einige seltene und neue Krustentiere in den Karstgewässern der Tschechoslowakei" (1956), il décrit une nouvelle forme (race géographique?), B.natans hrabei. Les différences signalées tiennent particulièrement à la structure des mandibules qui ont 7 dents. En présentant sa sous-espèce, Sterba remarque: "In der Mehrzahl stimmen diese Individuen mit der frühen allgemein erkannten Art Bathynella chappuisi Delachaux überein. $\|^{5}$ )

Kulhavy 1957, le second carcinologue qui a étudié les Bathynelles de Tchécoslovaquie, mentionne pour la première fois B.natans natans Vejd., e'est-à-dire une forme identique à celle de Vejdovsky. Bien qu'il s'agisse de la sous-espèce type, l'auteur relève que les individus étudiés ressemblent beaucoup à B. chappuisi Delachaux. La mandibule de ceux-ci se distinque nettement de celle des races d'Allemagne par ses 7 dents.

Récemment, Kulhavy (1961), puis Sterba (1963) annoncent la présence de B.natans stammeri Jakobi sur le territoire de la Tchécoslovaquie, le premier en faisant aussi connaître la structure de la mandibule de l'unique exemplaire de Vejdovsky.

Toutes les études publiées à la suite de l'ouvrage de Jakobí6), ainsi que celles parues en Tchécoslovaquie, ont comme point de départ la conception systématique de cet auteur, ou, plus précisément, le fait que Bathynella d'Allemagne appartient à la même espèce que Bathynella de Prague, de Bâle et de la Grotte de Ver.

Lorsque Delachaux (1919) décrit Bathynella chappuisi, à la fin de son étude, il pose la question qui n'aurait dû échapper à aucun spécialiste: «Bathynella natans Vejdovsky de Prague est-elle identi que à Bathynella natans de Bâle?». Cette question s'imposait, car Delachaux présentait une nouvelle espèce en se basant sur le matériel de Bâle et parce que B.chappuisi était la troisième redécouverte du genre. D'ailleurs, en continuant, il remarque: "C'est une question que l'on peut poser maintenant étant donné que la description de la première (Bathynella de Prague - N.A.) a

$\left.{ }^{5}\right)$ Sterba, 1956, p. 403.

6) Botoşaneanu et Damian, 1956; Botoşaneanu 1959; Mestrov, 1957; Delamare Deboutteville, 1961; Jankowskaja, 1964; Birstein et Ljovuschkin, 1964; Cvetkov et coll., 1965. 
été faite sur un seul exemplaire, mal conservé. Il serait donc d'un grand intérêt de retrouver également cette forme. $»^{7}$ )

On sait que la Bathynella trouvée par Chappuis à Bâle a fait l'objet de deux importants travaux, l'un par lui-même (1915), et l'autre par Calman (1917). C'est de la sorte qu'après 30 ans, enfin, nous trouvons dans la littérature spécialisée une description plus détaillée de B.natans Vejd., notamment d'un matériel qui a été attribué aussi bien par Chappuis que par Calman à B. natans Vejd. Bien que les deux auteurs aient eu à leur disposition l'unique exemplaire de Vejdovsky, ni l'un ni l'autre n'ont pu y voir rien de plus, par rapport à la description initiale. $\left.\left.{ }^{8}\right)^{9}\right)$

Depuis l'année où parût l'étude de Chappuis, le matériel de Bâle est tenu pour identique à celui de Prague, octroyant de la sorte des droits illimités à la découverte de Chappuis. En conséquence, les recherches ultérieures - sans faire les réserves nécessaires - ont attribué à Bathynella natans de Prague les individus de Bâle; les traits morphologiques de ces derniers étant attribués, à leur tour, à l'exemplaire de Prague.

En se fondant sur ce fait, Delachaux décrit B.chappuisi; Delamare Deboutteville et Chappuis attribuent à B.natans les exemplaires de Bâle et de Strasbourg; Jakobi publie son remarquable ouvrage. Ce dernier, en faisant la synonymie de B.chappuisi et B.natans, résoud l'une des plus délicates questions de la taxonomie du genre, mais en éliminant l'espèce de Delachaux, il ne peut sortir du dilemme: «Bathynella natans Vejdovsky de Prague est-elle identique à Bathynella natans de Bâle?

En tenant compte de tous les traits morphologiques utilisés dans la taxonomie du genre Bathynella, il était assez difficile de supposer qu'on pouvait en trouver d'autres, d'une aide plus utile. Les multiples

7) Delachaux, 1919, p. 18.

8) „Im Jahre 1900 erschien eine kurze Beschreibung des Tieres von W.T. Calman, der das Präparat Vejdovsky's von neuem untersuchte und mit Anaspides tasmaniae G. M. Thomson verglich. Er kam zu dem Schlusse, daß diese zwei Tiere ohne Zweifel verwandt sein müßten, doch konnte der englische Autor nichts Neues über Bathynella mitteilen." (Chappuis, 1914, p. 147).

$\left.{ }^{9}\right)$ Das Prager Präparat selbst ist im Laufe der Jahre stark geschrumpft, so daß man nur wenig Einzelheiten erkennen kann; da jedoch das gleiche Tier jetzt wieder gefunden worden ist, hat das Präparat nur noch historischen Wert." (Chappuis, 1914, p. 148.) 
considérations et raisonnements émis sur la chétotaxie des différents appendices excluaient dès le début la chance de réussite de n’importe quel essai de ce genre.

La synonymie de B.chappuisi Delachaux et B.natans Vejd. faite par Jakobi (1954) a eu pour premier résultat l'introduction d'une large variabilité des caractéristiques utilisées dans la systématique de natans. Dans cet ordre d'idées, Jakobi en étudiant la chétotaxie du basis des uropodes chez plusieurs populations (et aussi au cours du développement), affirme que B.natans Vejd. possède 4 à 8 épines. Ses recherches sur le péréiopode 8 femelle le conduisent à la conclusion que le rapport entre la longueur de l'exopodite et celle de l'endopodite varie dans de très larges limites. Par cette opinion il supprime l'un des caractères les plus nets que proposait Delachaux pour différencier les espèces. Sans discuter la valeur des autres résultats de Jakobi, nous sommes d'avis que certaines de ses opinions relatives à la large variabilité des caractères de B. natans Vejd., ne peuvent expliquer une série de résultats plus récents. En voici quelques exemples.

En consultant le travail de Delamare Deboutteville et Chappuis (1954), la note de Jankowskaja (1964), dans laquelle est présentée la sous-espèce B.natans issykkulensis, ainsi que celle de Birstein et Ljovuschkin (1964) qui décrivent B.natans ciscaucasica, on voit que, en dépit de cette aire géographique si étendue (Espagne-Tian Shan), tous les individus étudiés ont seulement 4 épines sur les basis de leurs uropodes.

Les recherches entreprises avec T. Gledhill sur deux populations de B.natans stammeri Jakobi provenant d'Angleterre et de Roumanie (Note sous presse), nous ont permis de constater, à l'encontre des affirmations du spécialiste allemand, la stricte constance de la plupart des caractéristiques utilisées dans la systématique du groupe. Ce fait revêt encore plus d'importance si on y ajoute le facteur espace: $2000 \mathrm{~km}$. de distance. Mais en laissant hors de cause ce point de vue, qui fait l'objet d'une autre étude, nous tenons à préciser qu'approximativement 60 individus étudiés ont présenté, sans aucune exception, seulement 4 épines sur le basis des uropodes. Les péréiopodes 8 de la femelle ont une constitution qui ne correspond pas à celle des exemplaires de Bâle, mais par contre rappellent ceux des individus de Binghöhle (Jakobi). Suivant notre opinion - qui n'est pas conforme à celle de Jakobi - en ce qui concerne la variabilité de B. natans Vejd., il existe une corrélation de certaines caractéristiques pouvant servir à séparer les sous-espèces.

Se rapportant au même trait (le nombre d'épines sur le basis des uropodes), Jakobi remarque, dans le cadre de certaines populations 
d'Allemagne, qu'il n'est point variable, tandis que dans d'autres il l'est extrêmement. Les populations de Möhrendorff, Binghöhle, UnterAsbach, Freiburg, présentant seulement 4 épines, se distinguent de celles de Bodenwöhr et d'Aschaffenburg dont le nombre d'épines varie entre 4 et 8 , ainsi que de celles de Bonn qui ont 7 à 8 épines.

Tout en tenant compte de ces quelques données, comment pourraiton expliquer le fait qu'un même caractère, constant chez des populations très éloignées au point de vue géographique, ait une extrême variabilité chez d'autres relativement rapprochées? Quels sont les facteurs qui pourraient expliquer ce double aspect de la variabilité de certains traits?

La chance d'avoir trouvé B.natans stammeri Jakobi dans une population de Roumanie (la grotte Peştera Lazului) qui présentait une "variabilité» du nombre des épines du basis des uropodes semblable à celle des individus de Bodenwöhr et Aschaffenburg, nous a permis d'expliquer les causes de cette large variabilité. Nous avons constaté qu'il s'agissait donc d'une population formée par deux unités taxonomiques différentes, à savoir B.natans stammeri Jakobi et B.natans Vejd. de Tchécoslovaquie. L'étude comparative de celles-ci a permis de relever le fait que la majorité des exemplaires ayant la mandibule semblable à celle de B.natans Vejd. de Tchécoslovaquie, avait 5 à 7 épines sur le basis des uropodes. Le péréiopode 8 des femelles, ainsi que celui de l'unique exemplaire mâle, nous ont donné la possibilité de remarquer une série de différences qui seront discutées partiellement dans cette note.

Ainsi, ce qui au début était pure supposition, commence à prendre jour de façon plus précise. Les données fournies par nos recherches plaident en faveur de la non identité entre le matériel de Bâle (Chappuis et celui de la grotte de Ver (Delachaux). Pour pouvoir le prouver, il nous fallait une caractéristique qui soit plus constante, plus démonstrative, par conséquent plus convaincante.

L'étude minutieuse du péréiopode 8 mâle, nous a offert l'élément recherché, car sa structure morphologique différencie nettement plusieurs formes. Notre attention a été dirigée sur cet appendice par le travail de Delachaux, qui a observé des différences évidentes entre les péréiopodes des individus de Bâle et ceux trouvés par lui. Nous citons: "Chez le mâle, par contre, cette paire de pattes joue le rôle d'un véritable pénis de forme compliquée, présentant un aspect très différent dans les deux espèces, tout en étant composée des mêmes éléments. Le conduit sexuel vient s'ouvrir au sommet d'un mamelon du premier article, protopodite. Chez Bathynella de Bâle, ce mamelon est dirigé 
en avant tandis que le reste de patte se dirige en arrière à angle droit par rapport à lui. " ${ }^{10}$ )

La sûreté avec laquelle il affirme son point de vue, nous a obligé à entreprendre une étude approfondie du péréiopode 8 mâle. Les différences que nous signalerons se rapportent à la region proximale du péréiopode, c'est-à-dire au mamelon même dont parle Delachaux. Si l'auteur de B. chappuisi se limite seulement à une observation comparative pas assez détaillée, nous, nous essayons de saisir la structure intime de cette zone du péréiopode, la seule qui soit caractéristique pour la détermination des espèces.

Dans ce qui suit, nous décrivons deux types de péréiopodes 8 màle, que nous avons dénommés le «type stammeri» et le «type natans».

\section{Le type stammeri (fig. $1 \mathrm{~B} ; 2 \mathrm{~A}-\mathrm{D} ; 6 \mathrm{~A}-\mathrm{C}$ )}

Présenté par Jakobi comme appartenant à B.natans Vejd. sensu Jakobi, nous l'avons décrit avec T. Gledhill de façon détaillée dans une autre note.

On distingue - outre les articles connus - dans la partie antérieure de la région basale du péréiopode 8 mâle (fig. $1 \mathrm{~B}$ ) une pièce rectangulaire caractérisée par un prolongement distal externe. Cette formation que Jakobi (1954) considère comme un basipodite, est la structure la plus représentative du péréiopode type stammeri. On doit préciser encore que la zone où se trouve cette formation (en fait, le mamelon dont parle Delachaux) n'est pas trop étendue, ne dépassant pas un tiers de la longueur de l'exopodite. En étudiant la région en question, où débouchent les canaux déférents, nous y trouvons les structures. suivantes.

Au premier plan de la partie antérieure, il y a une pièce que nous avons dénommée la plaque antérieure (fig. $2 \mathrm{~A}-\mathrm{a}$ et $6 \mathrm{~A}$ ). De forme rectangulaire, avec un prolongement dans l'angle inféroexterne, cette pièce ne dépasse pas la longueur du sympodite. Les limites interne, distale et externe sont évidentes, et bien contourées. Le bord interne à contour régulier présente une entaille chez tous les exemplaires; l'angle interne est distal, légèrement arrondi ; le bord distal légèrement concave passe dans un prolongement rhomboïdal; le bord externe est vertical. Il est à souligner que cette formation rhomboïdale n'est pas une épine - comme l'a affirmé Jakobi - mais un simple prolongement de la plaque antérieure.

Vue du côté latéral interne (fig. $2 \mathrm{C}-\mathrm{a}$ et $6 \mathrm{C}$ ) ou de celui externe (fig. $2 \mathrm{D}-\mathrm{a}$ et $6 \mathrm{~B}$ ), la plaque antérieure a une certaine épaisseur, sa

\footnotetext{
10) Delachaux, 1919, pp. 9-10.
} 
partie distale étant toujours terminée par une pointe : le prolongement rhomboïdal (vue de profil). Les trois épines visibles aussi en vue frontale, sont beaucoup plus évidentes en vue latérale, étant attachées à la plaque antérieure, avec les pointes dirigées en avant.

En ce qui concerne l'affirmation de Jakobi (1954), qui considère la plaque antérieure comme un basipodite, - en d'autres termes un article par lequel le péréiopode s'attache au segment du corps-nous précisons qu'elle n'est qu'une simple formation qui couvre la partie antérieure de la zone où débouchent les canaux déférents.

Le lobe interne (fig. $2 \mathrm{~A}-\mathrm{D}-\mathrm{b}$ et $6 \mathrm{~A}-\mathrm{C}$ ): Nous avons nommé ainsi le lobe situé sous la plaque antérieure au-dessus du bord interne du protopodite. Présentant la forme d'un cône à bout arrondi, cette formation est facile à voir de tous côtés. Vu du côté latéral interne, il paraît très massif, ayant la base à peu près entièrement délimitée par un sillon profond.

Le grand lobe (fig. $2 \mathrm{~A}-\mathrm{D}-\mathrm{c}$ ) semble un véritable article du péréiopode. A la partie externe il limite l'entier complexe structural; postérieurement il est disposé par dessous le sympodite.

Le petit lobe (fig. $2 \mathrm{~A}-\mathrm{D}-\mathrm{d}$ ) se place entre le grand lobe et le lobe interne. Si nous observons le péréiopode sur sa partie latérale interne, nous voyons que le petit lobe est bilobé et attaché par un pédoncule.

A une autre occasion (Note déjà mentionnée) nous avons dit qu'on ne peut pas préciser si toutes les formations décrites sont attachées directement au segment du corps ou par l'intermédiaire d'un autre article. Actuellement nous sommes d'avis que la seconde hypothèse est plus proche de la réalité. Par conséquent, le grand lobe n'est qu'un article - un coxopodite - qui s'interpose entre les formations décrites et le segment du corps. On ne distingue pas nettement les limites entre les différents lobes par rapport aux coxopodites, celles-ci nous sont suggérées par les petites concavités (vues de profil), qui existent sur chaque formation, à peu près au même niveau. Le basipodite, qui s'attache au coxopodite, près de la base de ce dernier (fig. 2 B), supporte à sa partie antérieure toutes les formations mentionnées.

\section{Le type natans (fig. $1 \mathrm{~A}, 3 \mathrm{~A}-\mathrm{D}, 7 \mathrm{~A}-\mathrm{D}$ )}

Delachaux fut le premier à présenter une esquisse (de profil) de ce péréiopode. D'une structure générale semblable à celle du type stammeri, il a dans sa partie frontale un lobe triangulaire (la plaque antérieure) d'une longueur égale ou dépassant celle de l'exopodite (fig. 1 A). Sous la partie interne de cette formation il existe un lobe cylindrique (le lobe interne) près duquel, vers la partie extérieure, il s'en trouve encore un, plus petit (le petit lobe). 
La plaque antérieure (fig. $3 \mathrm{~A}-\mathrm{D}-\mathrm{a}, 7 \mathrm{~A}-\mathrm{D}$ ), ainsi que nous l'avons dit, est de forme triangulaire, à bord externe incliné vers l'intérieure, tandis que l'interne, vertical, a une entaille nette. Le sommet arrondi est très proche de la limite interne du sympodite, étant par conséquent orienté vers l'intérieur du péréiopode. Vue de profil, la plaque antérieure (fig. 3 C, D-a, 7 C, D) est massive; sa partie distale paraît bilobée.

Le lobe interne (fig. $3 \mathrm{~A}-\mathrm{D}-\mathrm{b}, 7 \mathrm{C}$, D) se distingue de celui du type stammeri par sa forme cylindrique. Il n'est pas clairement délimité et ne dépasse jamais le bord distal de la plaque antérieure. Vu latéralement il est très ressemblant à la plaque antérieure; pas beaucoup plus petit et sans bord proximal évident.

Le petit lobe (fig. $3 \mathrm{~A}-\mathrm{D}-\mathrm{c}, 7 \mathrm{C}, \mathrm{D}$ ), plus difficilement observable à cause de ses dimensions réduites, est situé à la partie externe du sommet du lobe interne.

Dès le début il est nécessaire de souligner que la zone où débouchent les canaux déférents chez les deux types de péréiopodes est beaucoup plus complexe du point de vue morphologique. Il est nécessaire aussi de relever que les différences les plus évidentes entre ces deux types stammeri et natans -, s'observent de même dans cette zone, zone dont le rôle est d'importance majeure dans le processus de l'accouplement.

Malgré les différences observables chez les péréiopodes, l'aspect général et la disposition des différentes formations décrites prouvent la parenté de ces deux types, leur plan général d'organisation étant le suivant.

Dans la partie basale du péréiopode il y a un article massif, bien développé - le coxopodite - attaché au segment corporel portant sur lui la plaque antérieure, le lobe interne, le petit lobe ainsi que le basipodite. Sur ce dernier sont articulés l'endopodite - plus petit, et l'exopodite - mieux développé.

Outre le fait que les différences signalées suggèrent leur degré de parenté, elles justifient pour nous l'idée que les deux formes de péréiopodes appartiennent à deux types distincts. Cette opinion impose indubitablement la séparation en deux unités taxonomiques des individus auxquels les péréiopodes en question sont propres et qui, jusqu'à présent étaient classés dans l'espèce natans.

Il est peu probable que le matériel de Roumanie, qui possède le péréiopode type natans, n'appartienne pas à la vraie B.natans Vejd. Les données déjà connues ${ }^{11}$ ) ainsi que nos résultats, justifient pleinement l'idée que B.natans Vejd. est largement répandue en Roumanie.

11) Botoşaneanu et Damian, 1956; Botoşaneanu, 1959; Delamare Deboutteville, 1961. 
Elle représente d'ailleurs la majorité des populations considérées autrefois comme B. chappuisi Delachaux. ${ }^{12}$ )

La nette ressemblance entre les plaques antérieures du péréiopode 8 mâle des individus de Roumanie et de Tchécoslovaquie (Sterba, 1954), vient de confirmer l'appartenance à B.natans Vejd. de la plupart des populations trouvées dans les deux pays. Si nous y ajoutons la structure de la mandibule (fig. $5 \mathrm{~A}-\mathrm{E}$ ) qui ressemble fort à celle (fig. 5 F) de l'unique exemplaire de Vejdovsky (Kulhavy 1961), nous parvenons à éliminer toute trace de scepticisme concernant la présence de la véritable B.natans Vejd. en Roumanie.

En comparant les dessins du péréiopode 8 mâle de B.natans Vejd. de Roumanie (fig. $3 \mathrm{~A}-\mathrm{D}, 7 \mathrm{~A}-\mathrm{D}$ ) à celui présenté par Delachaux pour B. chappuisi (fig. 4 A), la ressemblance qu'on y trouve nous donne le droit d'intégrer le péréiopode de la population étudiée par Delachaux dans le type natans. Les autres caractéristiques morphologiques de ce que l'on avait désigné comme B.chappuisi, identifiées aussi chez les captures de Tchécoslovaquie et de Roumanie, prouvent que celui qui a retrouvé B.natans Vejd. fut Delachaux et non pas Chappuis. Les multiples affirmations des auteurs tchèques qui précisaient que $B$. natans Vejd., sensu Jakobi de Tchécoslovoquie, ressemble beaucoup à «B.chappuisi » trouvent aujourd'hui pleine satisfaction. Les considérations de Jakobi (1954) sur l'identité de l'espèce de Delachaux à celle de Vejdovsky, garde encore toute sa validité.

Cette identité établie entre le matériel de la Grotte de Ver et celui de Prague, ainsi que les différences entre le premier et la population de Bâle ${ }^{\mathbf{1 3}}$ ) (en dépit de l'ancien sens de la synonymie de Jakobi) plaident en faveur de la non identité de cette dernière avec B.natans Vejd. Parmi les différences signalées par Delachaux nous avons mentionné celles se rapportant au péréiopode 8 mâle. Il faut préciser que nous avons trouvé dans les quelques lignes déjà citées, appartenant à cet auteur, les plus précieuses indications concernant les péréiopodes 8 des deux populations.

En examinant plus attentivement le dessin de Delachaux (fig. 4 B), nous sommes d'avis que le péréiopode de la forme de Bâle tient du type stammeri et non du type natans. Cet avis se fonde sur la forme générale du mamelon (fig. 4 D) figuré par Delachaux pour B.natans Vejd. de Bâle et, particulièrement, sur l'aspect du sommet distal, qui peut être homologué au prolongement rhomboïdal de la plaque antérieure (fig. $2 \mathrm{C}, \mathrm{D}, 6 \mathrm{~B}, \mathrm{C}$ ). Les doutes qui pourraient encore subsister, assez naturels d'ailleurs, sont écartés par les nombreuses

\footnotetext{
12) Chappuis 1925 et 1944; Bartok 1944; Delamare Deboutteville et Chappuis, 1954. $\left.{ }^{13}\right)$ Delachaux, 1919.
} 
données se rapportant aux autres traits morphologiques. Ces derniers plaident nettement en faveur de l'identité spécifique du matériel de Chappuis et de Jakobi, qui fut présenté comme B.natans Vejdovsky.

Par conséquent, notre réponse au dilemme posé par Delachaux est catégoriquement négative: Bathynella de Bâle n'est pas identique à celle de Prague. Cette non identité ne se manifeste pas au niveau de catégories taxonomiques infraspécifiques, car il s'agit de deux espèces différentes, bien individualisées et nettement séparées par des traits morphologiques de premier ordre.

Si nous reconsidérons donc la sous-espèce B.natans stammeri Jakobi (classée par erreur dans l'espèce B.natans Vejd.) nous devons l'élever au rang d'espèce, nommée dorénavant Bathynella stammeri (Jakobi). Des quatre sous-espèces de Jakobi, nous avons choisi stammeri, car c'est la seule que nous ayons trouvée et pu étudier. La détermination de l'appartenance spécifique des 3 autres races d'Allemagne sera à la charge du spécialiste allemand ou de ceux qui les trouveront encore. Nous procédons de la sorte, notre conviction étant que dans certaines populations étudiées par Jakobi, il se trouvait un mélange d'espèces, ce qui justifierait sa synonymie.

En tenant compte de la structure des péréiopodes 8 mâles nous pouvons séparer les deux espèces faisant partie autrefois de B.natans Vejd. sensu Jakobi, de la façon suivante: - la première, Bathynella natans Vejdovsky caractérisée par le péréiopode 8 mâle à plaque antérieure triangulaire, fortement développée, de la même longueur que l'exopodite, le lobe interne cylindrique et le petit lobe très réduit;

- la seconde, Bathynella stammeri (Jakobi) différente de la première par sa plaque antérieure de forme rectangulaire et à prolongement dans l'angle distal interne, par le lobe interne à aspect de cône et par le petit lobe bilobé.

Il est certain qu'à ces traits morphologiques s'ajoutent d'autres caractéristiques des deux espèces, mais elles ne pourront être réparties rigoureusement à l'une des deux unités taxonomiques qu'après une révision des données et des captures déjà connues.

Enfin, voilà certaines considérations d'ordre zoogéographique à l'appui de notre opinion. Lorsque Jakobi a séparé les 4 races du territoire allemand, il a réussi à leurs attribuer des aires géographiques bien distinctes, élément essentiel pour l'existence de pareilles catégories taxonomiques. Mais la découverte de la «race» stammeri en Tchécoslovaquie ébranle le système de Jakobi, ce qui conduisit Sterba (1963) à supposer que stammeri appartient à un taxon inférieur ${ }^{\mathbf{1 4}}$ ). La

\footnotetext{
${ }^{14}$ ) voir aussi Delamare Deboutteville,1960.Mem.Mus.Civ.Stor. Nat.Verona, VIII : 297-299.
} 
découverte de la même espèce en Roumanie, nous donna la certitude qu'au moins stammeri ne peut être une race de B.natans; sa coexistence avec cette dernière dans la même population (grotte «Peştera Lazului») nous mène à la même conclusion que l'étude morphologique.

Si Bathynella de Bâle n'est pas synonyme de B.natans Vejd. comment pouvons-nous expliquer la validité de la synonymie de Jakobi dans la situation actuelle?

Au moment où Jakobi a considéré le matériel de Bâle comme identique à celui de la Grotte de Ver - la seule manière par laquelle il a pu établir cette synonymie - il n'était pas dans le vrai, car les populations en question n'appartenaient pas à la même espèce. Mais, pure coïncidence, en utilisant les dénominations B.natans Vejd. et B. chappuisi Delachaux, il a exprimé involontairement une vérité qui aujourd'hui est démontrée.

Par conséquent la synonymie de Jakobi a maintenant une base scientifique et une validité indubitable.

En revenant aux relations existantes entre B.natans Vejd. et $B$. stammeri (Jakobi) il nous faut préciser que les différences remarquées dans la structure du péréiopode 8 mâle nous donnent de précieuses indications sur leur degré de parenté prouvant que ces deux espèces ne sont pas très proches. Si on y joint leur large répartition, l'individualisation de certaines de leurs populations grâce à des traits caractéristiques importants, nous obligent à les classer en deux différents sous-genres. Dans le premier nous plaçons l'espèce natans - qui conservera le nom du genre -, dans le second - dénommé par nous Antrobathynella -, nous situons l'espèce stammeri.

En conclusion, ce qui jusqu'à présent a été considéré Bathynella natans Vejd. sensu Jakobi, a été séparé en deux espèces distinctes à la suite de toutes ces modifications - chacune d'entre elles appartenant à deux sous-genres, à savoir: Bathynella (Bathynella) natans Vejdovsky et Bathynella (Antrobathynella) stammeri (Jakobi).

Nous sommes convaincus que nos modifications correspondent aussi à une réalité phylogénétique. Dans cet ordre d'idées nous croyons que les deux espèces représentent deux lignées indépendentes à évolution parallèle, véritables vestiges ayant survécu depuis la période de la crise évolutive du groupe jusqu'à nos jours.

Les conséquences qu'impliquent les modifications suscitées doivent avoir comme suite la révision et la reclassification de tout le matériel déjà connu. Des données bibliographiques nous pouvons déduire que la plupart des captures qui après 1954 ont été tenues comme appartenant à l'espèce B.natans Vejd. sensu Jakobi, pourraient être réunies dans le cadre de l'espèce stammeri mais que de toutes ces populations 
font exception B.natans natans de Tchécoslovaquie, Bulgarie, France et Roumanie et B.natans scytica Botoşaneanu et Damian de Roumanie qui appartiennent à l'espèce natans; d'autre part il est possible d'attribuer à l'espèce de Vejdovsky d'autres captures considérées autrefois comme B.chappuisi Delachaux.

En continuant l'étude des péréiopodes 8 mâles chez différentes populations, on pourra identifier aussi d'autres espèces. C'est probablement le cas de Bathynella de Yougoslavie dont le péréiopode 8 mâle ressemble à celui de B.natans, mais qui est toutefois assez différent, selon les dessins de Karaman et de Mestrov.

Enfin, l'étude comparative des péréiopodes 8 mâles tirera au clair bon nombre de problèmes essentiels concernant la connaissance de Bathynella, comme serait par exemple celui des relations entre les espèces d'Europe et celles d'Asie.

$\mathrm{Au}$ fur et à mesure que les principaux problèmes de la taxonomie de Bathynella seront élucidés, on aura une base solide pour l'élaboration d'une révision mondiale de ce "degenerate member of the Syncarida ", ainsi que l'a qualifié Calman il y a un demi-siècle.

\section{RÉSUMÉ}

En étudiant minutieusement la structure du péréiopode 8 mâle de plusieurs populations de Bathynella de Roumanie et d'Angleterre, les différences de structures relevées ont permis d'identifier deux types de péréiopodes, bien individualisés, qu'on a nommé le type natans (fig. 1 A) et le type stammeri (fig. $1 \mathrm{~B}$ ).

En se basant sur les remarquables différences entre ces deux types, on a séparé de l'espèce B.natans Vejd. sensu Jakobi, B.stammeri (Jakobi), considérée par Jakobi comme sous espèce de l'espèce natans. Les populations de B.stammeri (Jakobi) étudiées, ont été collectées en Angleterre et en Roumanie.

Les faits exposés plus haut impliquent l'idée que la conception de Jakobi (1954), qui a dominé la taxonomie du groupe, ne correspond pas à la réalité, les deux unités taxonomiques - jusqu'à présent encadrées dans B.natans étant caractérisées par:

- Bathynella natans Vejdovsky - le péréiopode 8 mâle (fig. 1 A) à plaque antérieure triangulaire, fortement développée, de même longueur que l'exopodite (fig. $3 \mathrm{~A}-\mathrm{D}-\mathrm{a} ; 7 \mathrm{~A}-\mathrm{D}$ ), avec un lobe interne cylindrique (fig. $3 \mathrm{~A}-\mathrm{D}-\mathrm{b}$ ), et un petit lobe très réduit (fig. $3 \mathrm{~A}-\mathrm{D}$-c).

- Bathynella stammeri (Jakobi) diffère de la précédente par une plaque antérieure rectangulaire ayant un prolongement à son angle distal externe (fig. $2 \mathrm{~A}-\mathrm{D}-\mathrm{a} ; 6 \mathrm{~A}-\mathrm{C}$ ) un lobe interne conique (fig. $2 \mathrm{~A}-\mathrm{D}-\mathrm{b}$ ), et un petit lobe bilobé (fig. 2 A-D-d).

Si la structure générale des péréiopodes génitaux plaide, ainsi que les autres caractéristiques, pour une affinité visible, les différences observées dans la zone du péréiopode 8 mâle - où débouchent les canaux déférents limitent cette parenté. 
En joignant à ces différences la large répartition de ces espèces et l'individualisation de certaines de leurs populations (grâce à des traits caractéristiques importants), on est conduit à les classer en deux sous-genres différents. Dans le premier on met l'espèce natans - qui conservera le nom du genre -, et dans le second - dénommé Antrobathynella - on place l'espèce stammeri.

En conclusion, ce qui jusqu'à présent a été considéré comme Bathynella natans Vejd. sensu Jakobi, a été séparé en deux espèces distinctes - à la suite de toutes ces modifications - chacune d'entre elles appartenant à deux sous genres différents, à savoir : Bathynella (Bathynella) natans Vejdovsky et Bathynella (Antrobathynella) stammeri (Jakobi).

Cette étude démontre que la synonymie faite par Jakobi - B.chappuisi synonyme de B.natans - est parfaitement valable dans les conditions ainsi créées - Delachaux (1919) ayant retrouvé B.natans Vejdovsky et non Chappuis (1914). Le matériel trouvé par ce dernier à Bâle (1914) appartienttenant compte des affinités - à B.stammeri (Jakobi) car il est différent, tant des exemplaires trouvés dans la Grotte de Ver (Delachaux, 1919), que de ceux de Prague (Vejdovsky, 1882).

\section{S U M MARY}

After a minute study of the structure of the 8 th male pereiopod in some Bathynella populations from Rumania and England, the structure differences which were found allowed to identify two well individualized kinds of pereiopods; they were named type natans and type stammeri.

Taking into account the striking differences between these two types, B. stammeri (Jakobi), which since 1954 is considered to be a subspecies of the natans species, was separated out of the species B.natans sensu Jakobi (1954). The populations understudied were collected in England and $\mathrm{Ru}$ mania, their minute study being the object of an other note, collaboration with T. Gledhill.

The facts led to the conclusion that Jakobi's opinion (1954), which dominated the taxonomy of this group, doesn't entirely correspond to the reality, the two taxonomical units being characterized as follows:

- Bathynella natans Vejdovsky, characterized by the 8th male pereiopod (fig. 1 A) with a triangular, well developped anterior plate (fig. 3 A-D-a; $7 \mathrm{~A}-\mathrm{D}$ ), of the same length with the exopodit, a cylindrical internal lobe (fig. 3 A-D-b,) and a little lobe (fig. $3 \mathrm{~A}-\mathrm{D}-\mathrm{c}$ ) of a reduced size;

- Bathynella stammeri (Jakobi) differing from the first with respect to the anterior plate (fig. $2 \mathrm{~A}-\mathrm{D}-\mathrm{a} ; 6 \mathrm{~A}-\mathrm{C}$ ) which is rectangular in shape and has a prolongation in the distal outer angle, to the conelike internal lobe (fig. $2 \mathrm{~A}$ $\mathrm{D}-\mathrm{b}$ ), and to the little lobe (fig. $2 \mathrm{~A}-\mathrm{D}-\mathrm{d}$ ) which is twolobed in this case.

After discussion of the relationship between B.natans Vejd. and $B$. stammeri (Jakobi) it is shown that differences observed in the 8th male pereiopod structure give important indications about the above species to the effect that they are not very closely related. If one takes into account also their wide spreading area, and the individualisation of some populations - due to important, characteristic traits - we are obliged to classify them into two different sub-genera. In the first one, the species natans is included - which will keep by this way the very name of the genus, and in the second, termed here Antrobathynella, the species stammeri. 
In conclusion, what was till now considered as Bathynella natans Vejdovsky sensu Jakobi, was divided into two distinct species - each of them pertaining to two different sub-genera, that is: Bathynella (Bathynella) natans Vejdovsky and Bathynella (Antrobathynella) stammeri (Jakobi).

It is demonstrated that the synonymy Jakobi made between B.chappuisi and $B$.natans is perfectly true under the new conditions too, because it was Delachaux (1919) who rediscovered B.natans Vejd., not Chappuis (1914). The material found by Chappuis in Basel (1914) appears to pertain to $B$. stammeri (Jakobi) differing both from the individuals from the Grotte de Ver (Delachaux, 1919) and from Prague (Vejdovsky, 1882).

\section{BIBLIOGRAPHIE}

Barток, P. A. - 1944: A. Bathynella chappuisi fejlödes morphologiaja Acta Sci. Math. Nat. Univ. Francisco-Josephina, Koloszvar, XXI: 1-46.

Birstein, J. A. and Luovuschin, S. I.,-1964: A new subspecies of Bathynella natans Vejd.(Crustacea, Bathynellacea) from subterranean waters of the Ciscaucasia. Zool. Jur., XLIII, 11, pp. 1719-1722.

Botoşaneanu, L. et Damian, A. - 1956: Bathynella (Crust. Syncarida) dans l'eau des conduites de Bucarest et de Constanza. Acta Soc. Zool. Bohemoslovenicae, XX, 4: 358-363.

- 1959: Bathynellacea. Fauna R. P. R., Crustacea, IV, 5: 1-34.

Brtek, J.- 1964: Funde von Bathynella natans Vejd. und Parabathynella stygia Chapp. im Gebiet von Horna Nitra (Mittelslowakei). Ac. Rer. Natur. Mus. Nat. Slov. Bratislava, X, 34-44.

Calman, W. T. - 1917: Notes on the morphology of Bathynella and some allied Crustacea. Quart. J. Micr. Sci. LXII : 489-514.

Chappuis, P. A. - 1914: Über die systematische Stellung von Bathynella natans Vejdovsky. Zool. Anz., XLIV: 45-46.

- 1915: Bathynella natans und ihre Stellung im system. Zool. Jahrb., XL: $147-176$.

— -1924-1925: Sur les Copépodes et les Syncarides des eaux souterraines de Cluj et des monts Bihor. Bull. Soc. de stiinte din Cluj, II, 4: 157-158.

- 1939: Úber Bathynella und Parabathynella. Vestnik Ceske Zool. Spol. v. Praze, VI-VII : 120-132.

- - 1944: Die Grundwasserfauna der Körös und des Szamos. Math. Term. Közl. Vonat. Mag. Tudomanyos Akademia, XL, 2: 1-44.

- - 1948: Copépodes, Syncarides et Isopodes des eaux phréatiques de Suisse. Rev. Suisse de Zool., LV, 30: 549-566.

Cretrov, L., Angelov, A. et Petrova, A. - 1965: Bathynellidae (Crustacea, Syncarida) de la Trace. Izv. Zoolog. it pri BAN.

Delachaux, Th. - 1919: Bathynella chappuisi n. sp. une nouvelle espèce de Crustacé cavernicole. Bull. Soc. Neuchâteloise Sci. Nat., XLIV: 1-20.

Delamare Deboutteville, Cl. - 1953: Présence du genre Bathynella dans le cours souterrain du Tech. Vie et Milieu, IV: 4.

- 1953: Au sujet des Bathynella de Frace. Vie et Milieu, IV, 4.

— 1961: Nouvelles récoltes de Syncarides et complément systématiques. Ann. spéléol., XVI, 2. 
- et Ghappuis, P. A. - 1954: Les Bathynelles de France et d'Espagne avec diagnoses d'espèces et de formes nouvelles. Arch. de Zool. exp. et gén., XCI, I : 51-73.

EFord, I. E. - 1959: Rediscovery of Bathynella chappuisi Delachaux in Britain. Nature, Lond. CLXXXIV: 558-559.

Gledhill, T. and Driver, D. B. - 1964: Bathynella natans Vejdovsky (Crustacea: Syncarida) and its occurrence in Yorkshire. The Naturalist, July-September: 104-106.

Hertzog, L. - 1936: Crustaceen aus unterirdischen Biotopen des Rheintales bei Straßburg. I. Mitteilung. Zool. Anz., CXIV: 271-279.

JAN KowskaJA, A. I. - 1964: Relict Crustaceans of Costal bottom waters of the Lake Issyk-Kul (North Tien-Shan). Zool. Jur., XLIII, 7: 975-986.

Jаков , H. - 1954: Biologie, Entwicklungsgeschichte und Systematik von Bathynella natans Vejd. Zool, Jahrb. LXXXIII, 1/2: 1-62.

Karaman, S. - 1934: Die Bathynelliden von Skoplje. Mitt. Höhlen u. Karstforsch. 26-30.

- - 1954: Über die Bathynelliden Jugoslaviens. Fragm. Balcan. Mus. Maced. scient. natur., I, 8: 70-78.

KIEFER, F. - 1925: Über einen neuen Fundort von Bathynella (Syncarida). Zool. Anz. Leipzig, LXIV: 101-102.

- 1928: Zur Kenntnis der geographischen Verbreitung und von Bathynella chappuisi Delachaux (Syncarida). Zool. Anz., LXXVIII: 123125.

Kulhavy, V. - 1957: Der zweite Fund von einer Bathynella in Böhmen und einige Bemerkungen zur Systematik der tschechoslowakischen Bathynellen. Acta Soc. Zool. Bohemoslovenicae, XXI, 3: 284-287.

- - 1961. Über das Vorkommen der west- und osteuropäischen Elemente in der Crustaceenfauna der böhmischen unterirdischen Gewässer. Vestnik Cs. spol., zool., XXV: 297-301.

Maitland, P. S. - 1962: Bathynella natans new to Scotland. Glasg. Nat. XVIII : $175-176$.

Mestrov, M. - 1957: Bathynellacea aus dem Grundwasser von Zagreb. Biol. Glasnik 10.

Nicholls, A. G. - 1946: Syncarida in relation to the interstitial habitat. Nature. Lond., CLVIII : 943-936.

Pony, E. - 1957 : Neue Bathynelliden aus Ungarn. Acta Zool. Acad. Scient. Hung., III, 1-2: 171-177.

Spooner, G. M. - 1961: Bathynella and other interstitial Crustacea in Southern England. Nature, CXC: 104-105.

Sterba, O. - 1954: Über Bathynella chappuisi Delachaux und andere Krebstiere der Brünner Brunnen. Casop. Moravsken. Musea Brne. Vedi prirod, X X XIX: 164-173.

— 1955: Beitrag zur Kenntnis der Krebstierefauna einiger Karstgewässer in der Slowakei. Spisy vydavane Prir. fak. Masarykovy Univ. v. Brne, CCGLXIV: 1-6. 
Sterba, O. - 1956: Einige seltene und neue Krustentiere in den Karstgewässern der Tschechoslowakei. Biologia, Bratislava, XI: 385-402.

— - 1961: Krebstiere der Grundwasser des oberen Flußlaufes des Laborek. Biologia, Bratislava, XVI : 821-825.

_- 1963: Bemerkung über zwei bemerkenswerte Funde von Bathynellidae (Crustacea, Anaspidacea) in der Tschechoslowakei. Zoolog. listy XII: 261-262.

Тӧвӧк, P. - 1947: The occurrence of Bathynella in the Budapest aqueduct. Fragm. Faunistica Hung., X.

— - 1951: Quelques nouvelles écrevisses caractéristiques pour l'eau souterraine concernant la faune de la Hongrie. Acta Biol. Acad. Scient. Hung., II, 1-3: 281-282.

Vejdovsky, Fr. - 1882: Thierische Organismen der Brunnenwässer von Prag. Prag. 1-70.

Wegelin, R. - 1961 : Über zwei eucavale Crustaceen aus dem interstitiellen Grundwasser der Leipziger Umgebung. Int. Revue ges. Hydrobiol., XIL, 1: 162-172.

\section{EXPLICATIONS DES PLANGHES $29(1)-35(7)$ PLANCHE 29 (1)}

Fig. 1: A: Bathynella (Bathynella) natans Vejdovsky, péréiopode 8 mâle; B : Bathynella (Antrobathynella) stammeri (Jakobi), péréiopode 8 mâle.

\section{PLANCHE $30(2)$}

Fig. 2: Région basale du péréiopode 8 mâle de Bathynella (Antrobathynella) stammeri (Jakobi). A : vue frontale; B : vue postérieure; C: vue latérointerne; D: vue latéro-externe. a: plaque antérieure; b: lobe interne; c: grand lobe (coxopodite); d: petit lobe; e: basipodite.

\section{PLANCHE 31 (3)}

Fig. 3: Bathynella (Bathynella) natans Vejd. Région basale du péréiopode 8 mâle de Bathynella (Bathynella) natans Vejd. A: vue frontale; B: vue postérieure; C: vue latéro-interne; D: vue latéro-externe. a: plaque antérieure; $\mathrm{b}$ : lobe interne; $\mathrm{c}$ : petit lobe; $\mathrm{d}$ : sympodite.

\section{PLANCHE $32(4)$}

Fig. 4: Péréiopode 8 mâle de: A: Bathynella (Bathynella) natans Vejd. de la Grotte de Ver (d'après Delachaux), B et C Bathynella de stammeri (Jakobi) de Bâle (B: d'après Delachaux et C: d'après Calman).

\section{PLANGHE $33(5)$}

Fig. 5: Structure de la partie masticatrice de la mandibule de Bathynella natans Vejd. trouvée en: A - Vallée «Iadului» (Transylvanie, Roumanie); B: Grotte "Peştera Tismana" (Olténie, Roumanie); C: Grotte "Peştera Lazului» (Olténie, Roumanie); D: Vallée de la Néra (Banat, Roumanie); E: Vallée du Motru Mare (Olténie, Roumanie); F : Prague (l'exemplaire de Vejdovsky, d'après Kulhavy 1961). 


\section{PLANCHE 34 (6)}

Fig. 6: Péréipode 8 mâle de Bathynella (Antrobathynella) stammeri (Jakobi): A: vue frontale; B: vue latéro-externe; $\mathrm{C}$ : vue latéro-interne.

Fig. 7: Péréiopode 8 mâle de Bathynella (Bathynella) natans Vejd. A et B: vue frontale; $C$ et $D$ : vue latéro-externe.

Fig. 8: Bathynella (Bathynella) natans Vejd. Aspect général de la femelle.

$$
\text { PLANCHE } 35 \text { (7) }
$$

Fig. 9: Bathynella (Antrobathynella) stammeri (Jakobi). Aspect général de la femelle. 


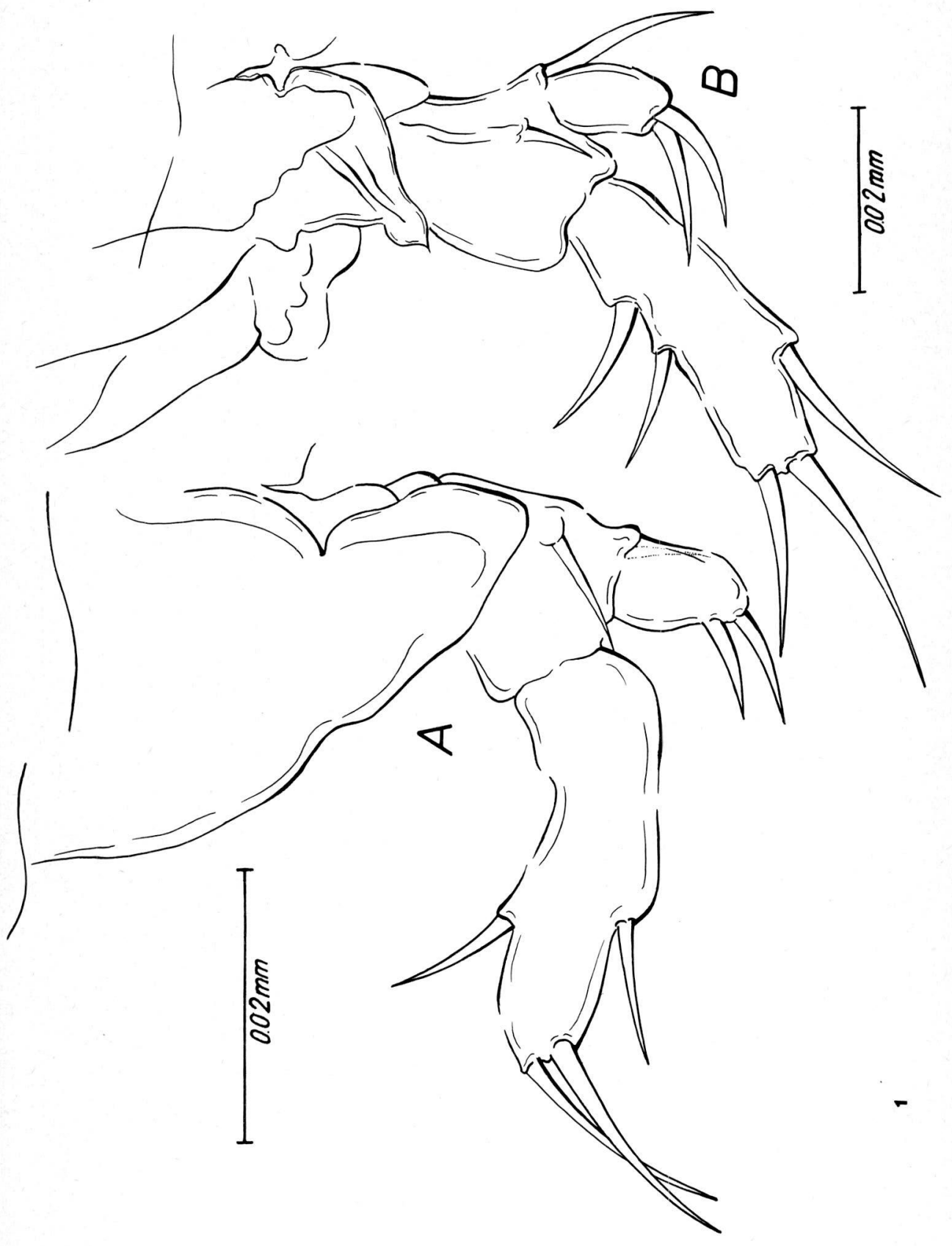



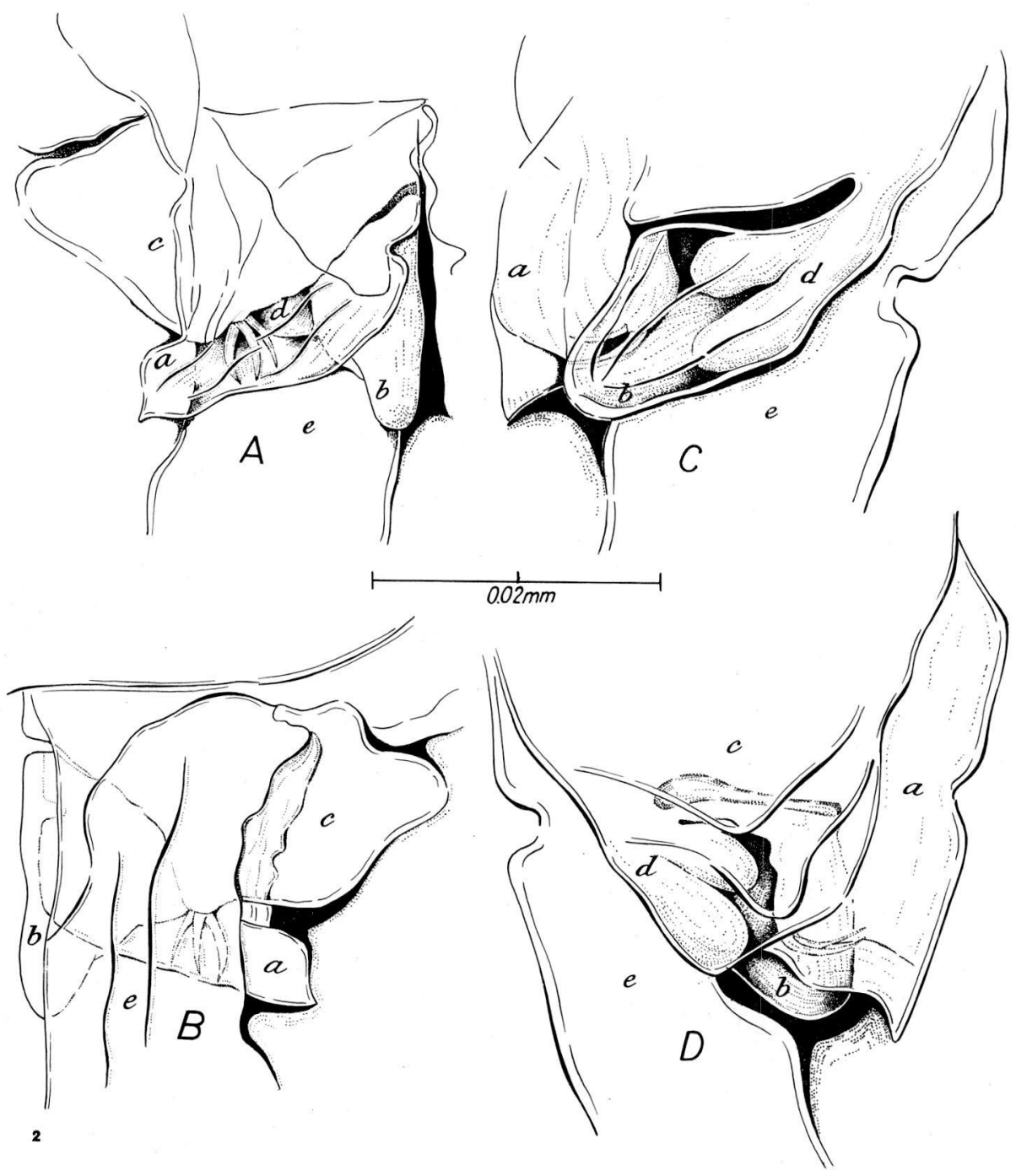

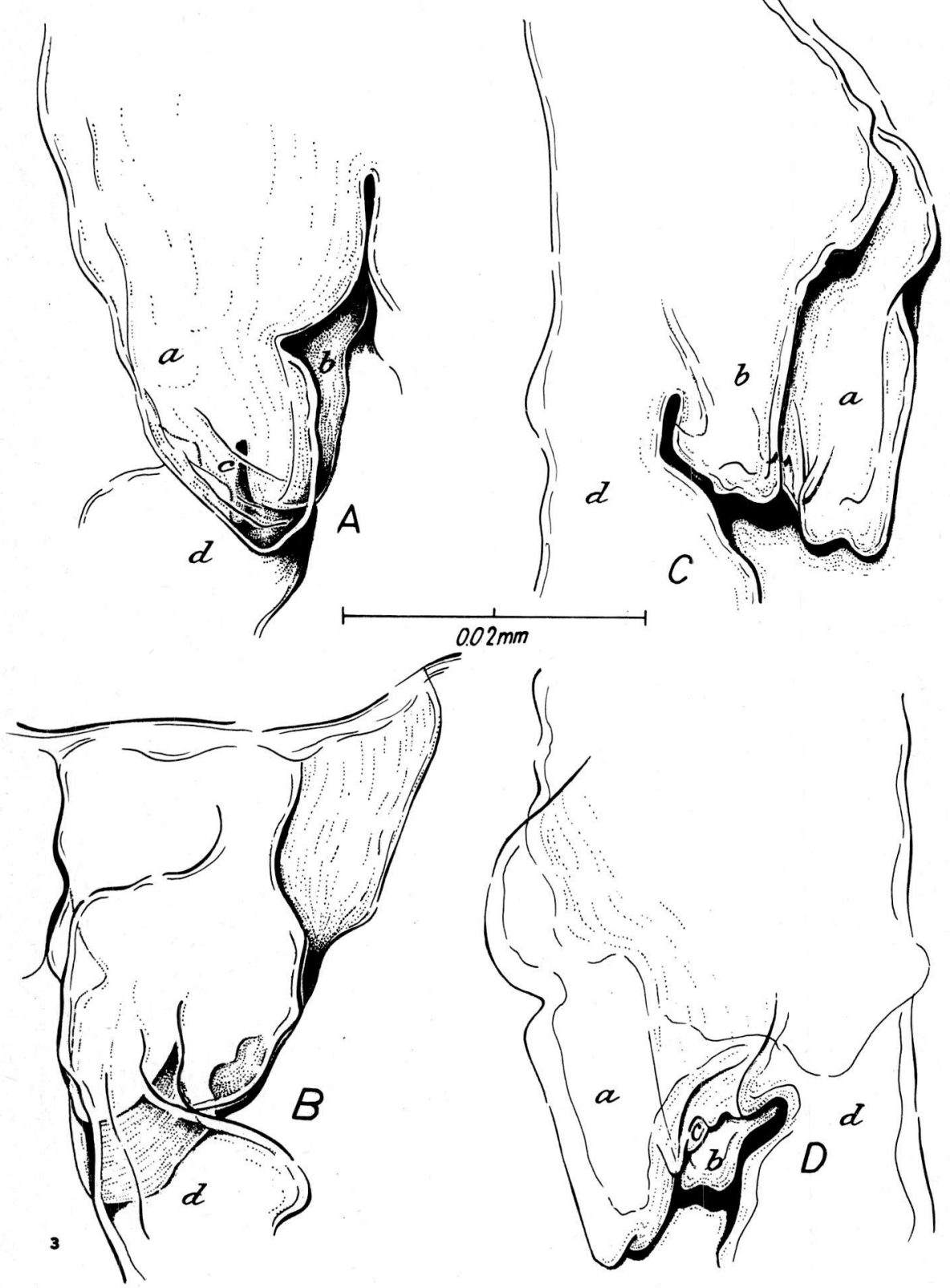

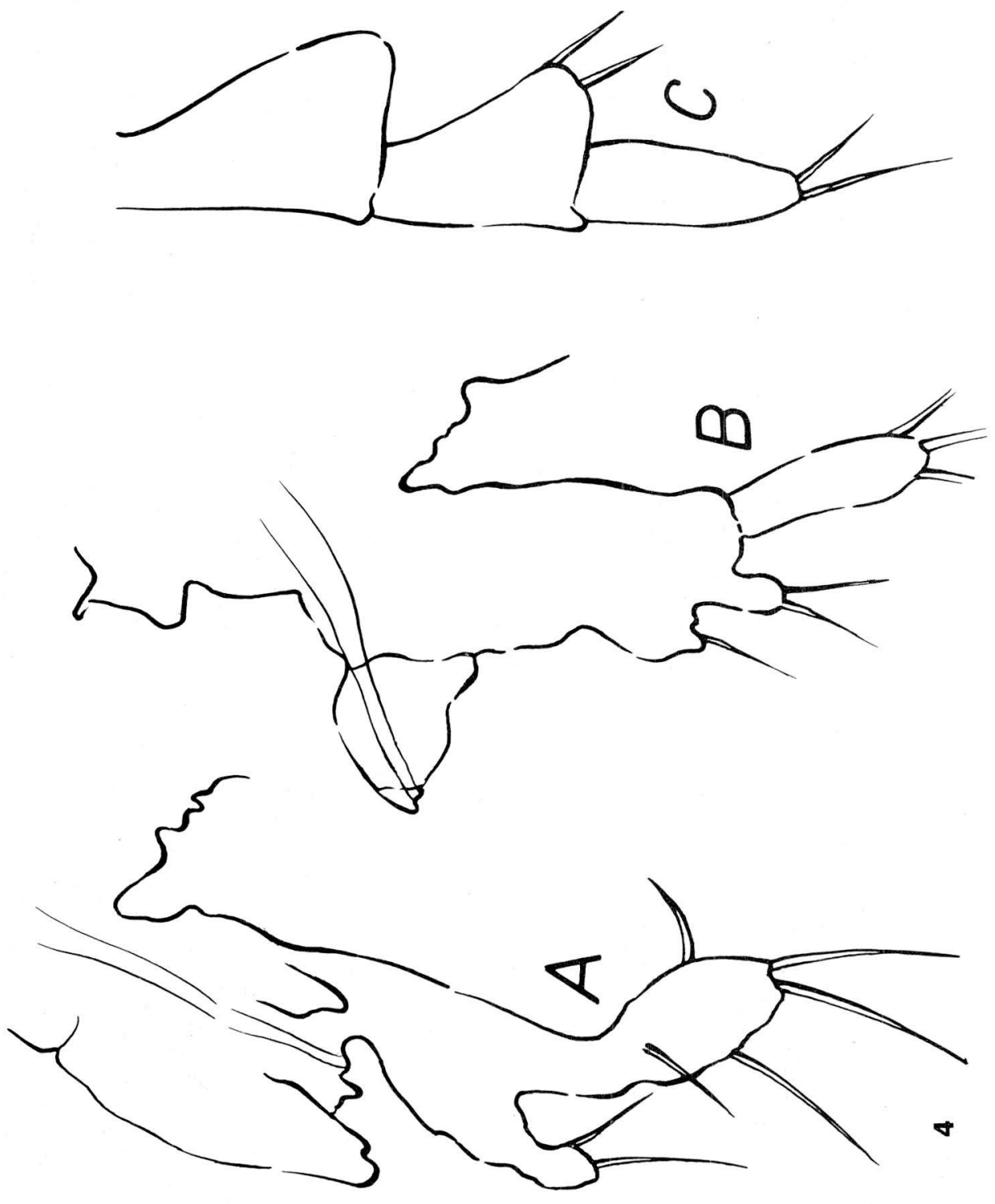

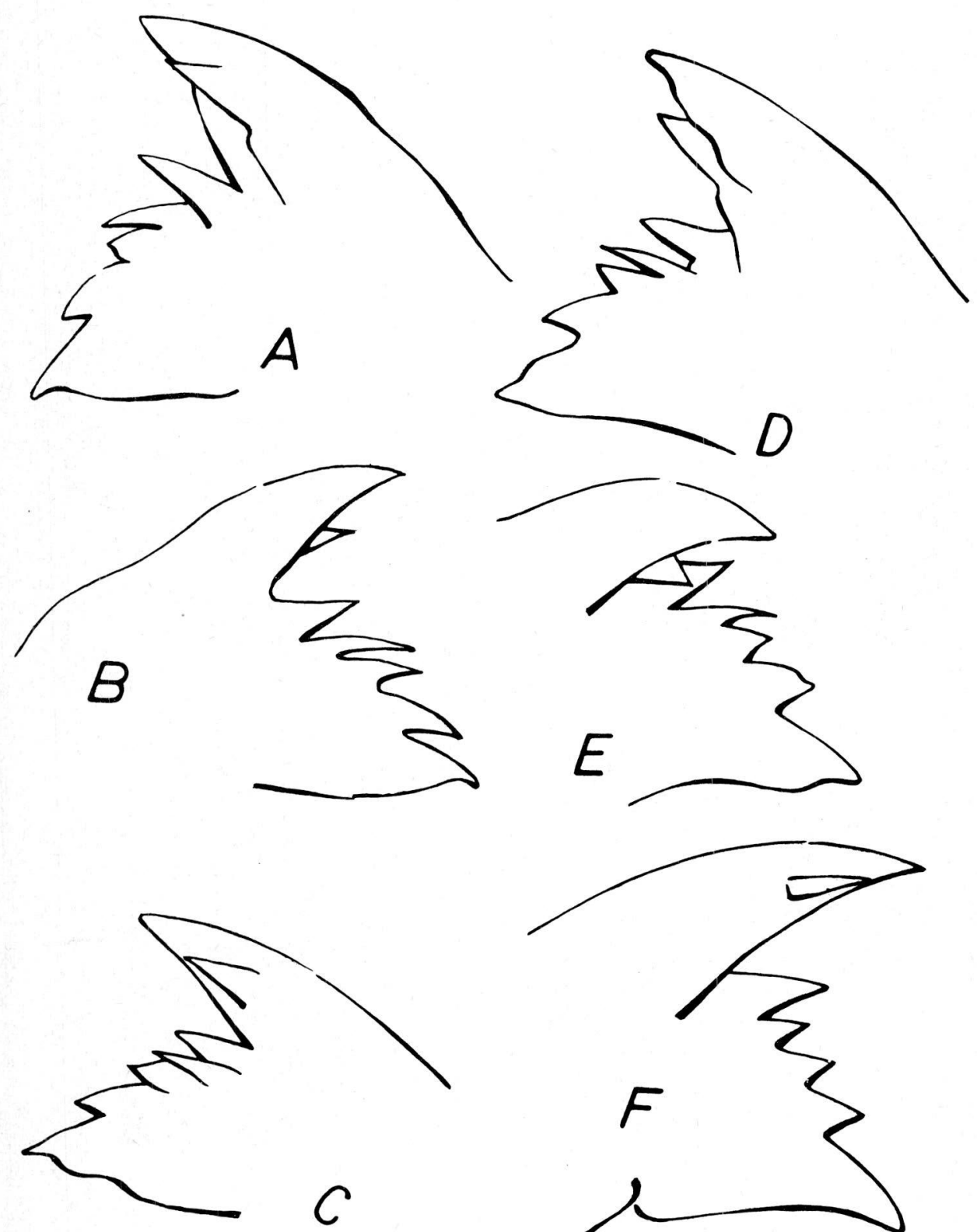

5
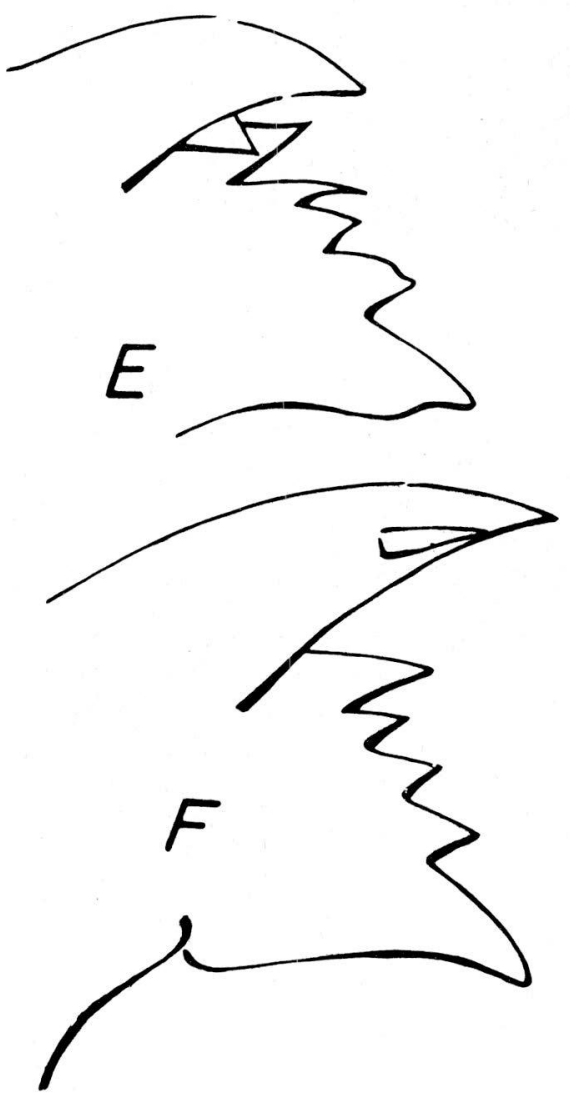

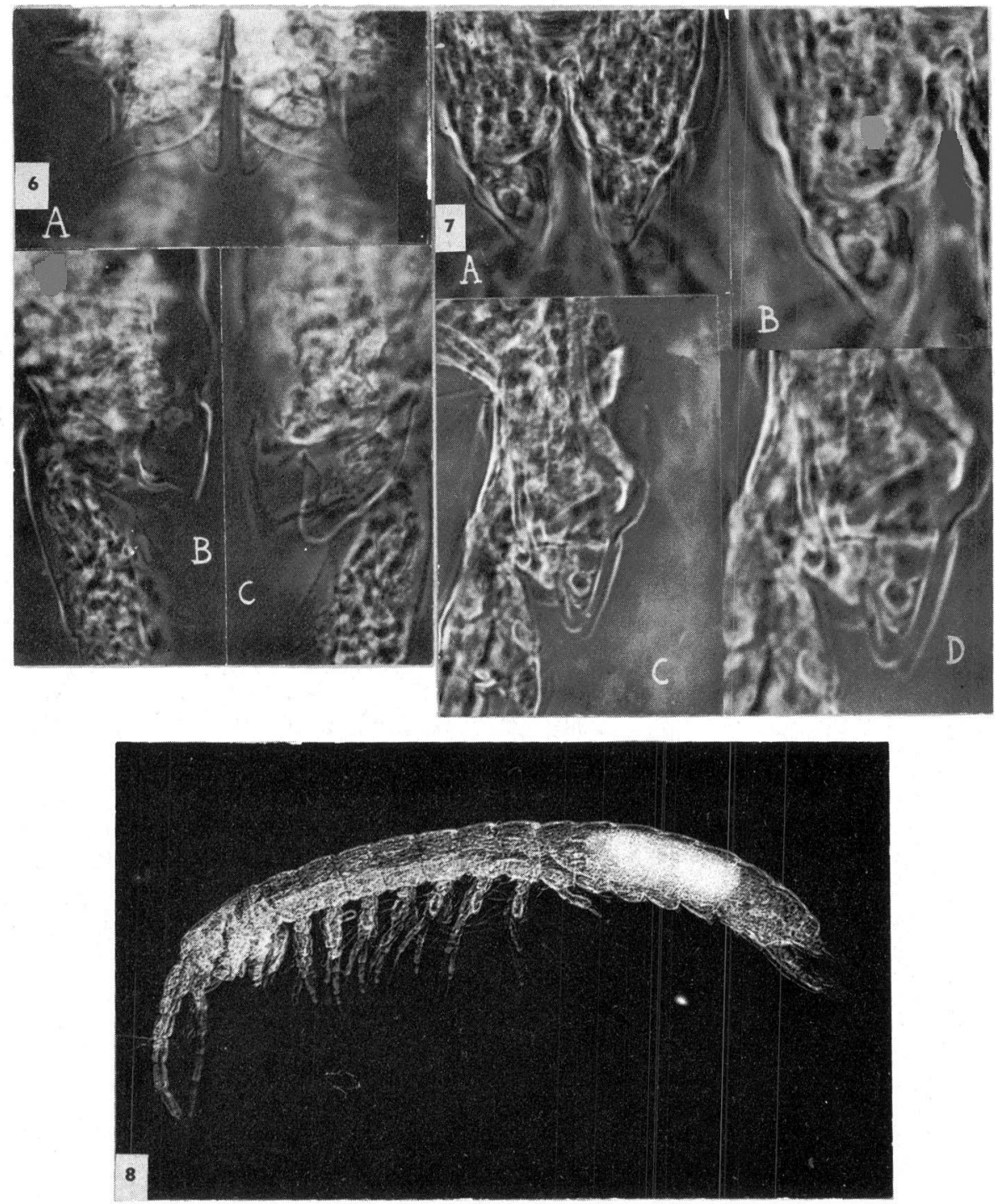


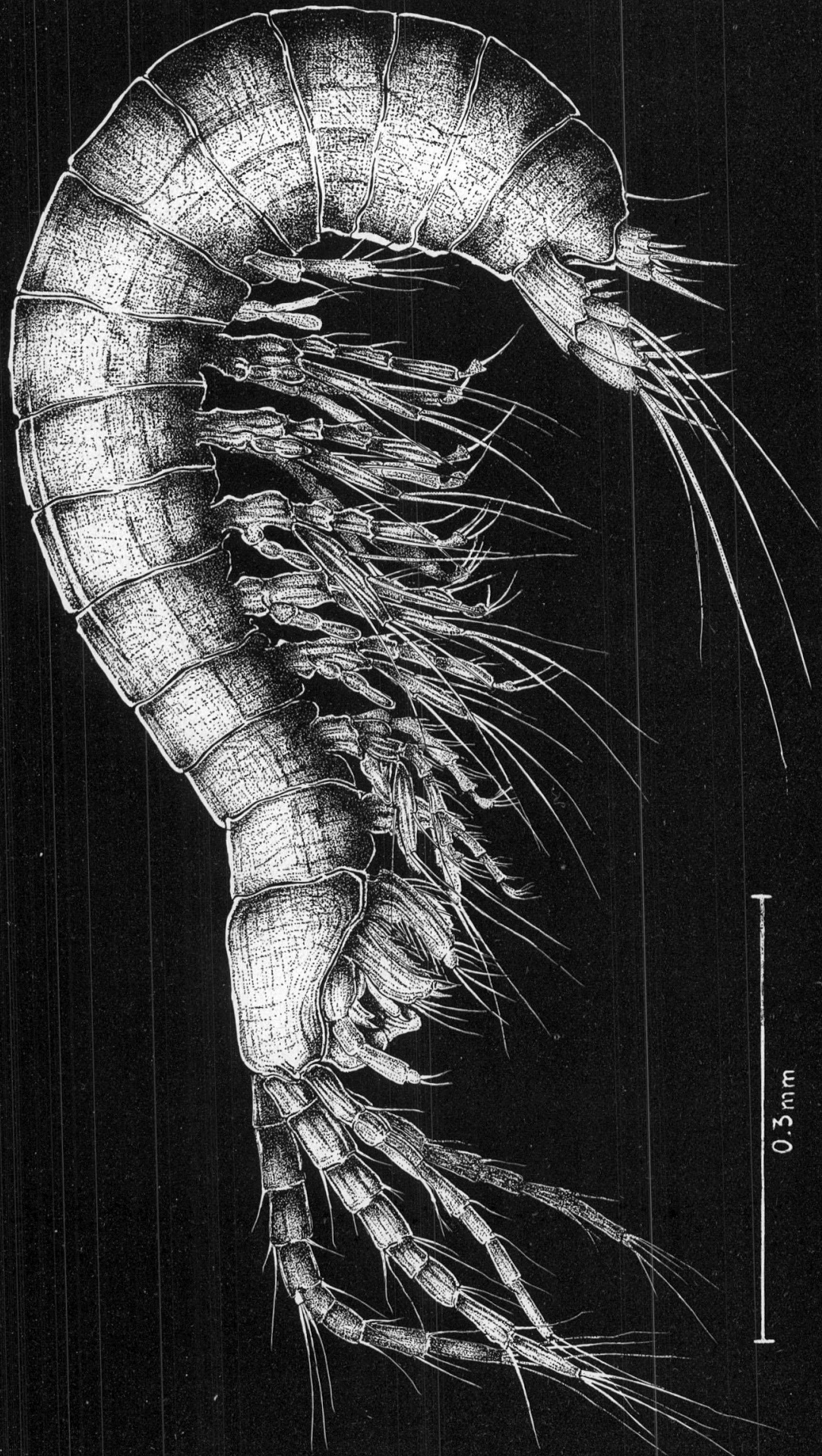

